\title{
SUBDIFFERENTIAL CONDITIONS FOR CALMNESS OF CONVEX CONSTRAINTS*
}

\author{
R. HENRION ${ }^{\dagger}$ AND A. JOURANI ${ }^{\ddagger}$
}

\begin{abstract}
We study subdifferential conditions of the calmness property for multifunctions representing convex constraint systems in a Banach space. Extending earlier work in finite dimensions [R. Henrion and J. Outrata, J. Math. Anal. Appl., 258 (2001), pp. 110-130], we show that, in contrast to the stronger Aubin property of a multifunction (or metric regularity of its inverse), calmness can be ensured by corresponding weaker constraint qualifications, which are based only on boundaries of subdifferentials and normal cones rather than on the full objects. Most of the results can be immediately interpreted in the context of error bounds.
\end{abstract}

Key words. calmness, multifunctions, convex systems, error bounds, constraint qualifications

AMS subject classifications. 90C31, 26E25, 49J52

PII. S1052623401386071

1. Introduction. Following [24, p. 399], a multifunction $M: Y \rightrightarrows X$ between metric spaces $X, Y$ is calm at some point $(\bar{y}, \bar{x})$ of its graph if there exist neighborhoods $\mathcal{V}, \mathcal{U}$ of $\bar{y}, \bar{x}$, respectively, and some $L>0$ such that the corresponding distance functions satisfy

$$
d(x, M(\bar{y})) \leq L d(y, \bar{y}) \quad \forall x \in M(y) \cap \mathcal{U}, \forall y \in \mathcal{V} .
$$

With $\mathcal{U}:=X$, calmness reduces to the upper Lipschitz property of multifunctions, introduced by Robinson [23]. Obviously, calmness is also weaker than the well-known Aubin property of multifunctions

$$
d\left(x, M\left(y^{\prime}\right)\right) \leq L d\left(y, y^{\prime}\right) \quad \forall x \in M(y) \cap \mathcal{U}, \forall y, y^{\prime} \in \mathcal{V} .
$$

(In particular, $M(y)=\emptyset$ for $y$ close to but different from $\bar{y}$ is possible under calmness but violates the Aubin property.) Calmness plays a key role in many issues of mathematical programming like optimality conditions, error bounds, or stability of solutions. The focus of this paper will be on multifunctions defined by convex systems in a Banach space $X$ like

$M(y):=\{x \in C \mid f(x) \leq y\} \quad$ or $M(y):=\{x \in X \mid d(x, C)+d(x, D) \leq y\} \quad(y \in \mathbb{R})$, $(1.3)$

where $C, D \subseteq X$ are closed, convex subsets and $f$ is convex. Of course, writing down the calmness property (1.1) for the first system considered in (1.3) immediately yields the existence of a local error bound for $f$ with respect to the set $C$. Hence all results obtained for this first part have an immediate link to the context of error bounds, which are extensively studied in the literature (e.g., [5], [15], [14], [16], [17], [19]). The aim of this paper is to derive dual (i.e., formulated in terms of the subdifferential and normal cone) conditions for calmness of the systems in (1.3) which are weaker than

\footnotetext{
* Received by the editors March 2, 2001; accepted for publication (in revised form) March 16, 2002; published electronically October 1, 2002.

http://www.siam.org/journals/siopt/13-2/38607.html

$\dagger$ Weierstrass Institute, Mohrenstrasse 39, 10117 Berlin, Germany (henrion@wias-berlin.de).

¥Université de Bourgogne, Département de Mathématiques, Analyse Appliquée et Optimisation, BP 47870, 21078 Dijon, France (jourani@u-bourgogne.fr).
} 
the well-known Slater-type conditions implying the stronger Aubin property (1.2) of $M$ (or, equivalently, the metric regularity of $M^{-1}$ ).

If $M$ is a polyhedral multifunction, then it is automatically calm (see [23]). Apart from this special class, certain conditions have to hold true in order to ensure calmness, and it seems natural to characterize these conditions in terms of well-known objects from nonsmooth analysis such as (co-) derivatives, (sub-) differentials, or tangent or normal cones. Similar characterizations have been successfully established for the stronger Aubin property. In finite dimensions, for instance, (1.2) is equivalent to each of the following two conditions, described by Mordukhovich [18] and Aubin (see, e.g., [1] and [6, Corollary 1.19] for necessity), respectively:

$$
\begin{aligned}
D^{*} M(\bar{y}, \bar{x})(0) & =\{0\}, \\
\exists \alpha, \beta>0: B(0,1) & \subseteq D_{-} M^{-1}(x, y)(B(0, \alpha)) \quad \forall x, y \in \operatorname{Gph} M \cap B((\bar{x}, \bar{y}), \beta) .
\end{aligned}
$$

Here, $D^{*}$ and $D_{-}$refer to Mordukhovich's coderivative and to Aubin's contingent derivative, respectively, while $B$ refers to appropriate closed balls. As coderivatives relate to normal cones while derivatives are associated with tangent cones, the first criterion above is of dual nature and the second one is of primal nature. The question arises of whether the criteria above can be modified appropriately to characterize the weaker calmness property (1.1) rather than (1.2). A primal criterion of calmness was found in [9, Proposition 2.1] (sufficiency) and [10, Proposition 4.1] (necessity):

$$
D M(\bar{y}, \bar{x})(0)=\{0\} .
$$

Note that (1.6) immediately enforces the isolatedness of $\bar{x}$ in $M(\bar{y})$ because a sequence $x_{n} \rightarrow \bar{x}, x_{n} \in M(\bar{y}), x_{n} \neq \bar{x}$ would generate a nontrivial tangent vector $(0, \xi)$ to $\operatorname{Gph} M$ at $(\bar{y}, \bar{x})$, whence a contradiction $0 \neq \xi \in D M(\bar{y}, \bar{x})(0)$ to (1.6). Consequently, a reduced version of calmness (also called calmness on selections) is equivalently characterized by (1.6). A dual characterization of calmness in the broader sense of (1.1) was given in [7] for the special case of finite-dimensional multifunctions

$$
M(y):=\{x \in C \mid g(x)+y \in D\},
$$

where $C \subseteq \mathbb{R}^{p}, D \subseteq \mathbb{R}^{m}$ are closed subsets and $g: \mathbb{R}^{p} \rightarrow \mathbb{R}^{m}$ is locally Lipschitz. In this special case, Mordukhovich's criterion (1.4) for the Aubin property takes the form

$$
\bigcup_{y^{*} \in N_{D}(g(\bar{x})) \backslash\{0\}} D^{*} g(\bar{x})\left(y^{*}\right) \cap\left(-N_{C}(\bar{x})\right)=\emptyset,
$$

where $N$ refers to Mordukhovich's normal cone. It was shown in [7] that under mild assumptions, calmness is implied by the weaker condition

$$
\bigcup_{y^{*} \in N_{D}(g(\bar{x})) \backslash\{0\}} D^{*} g(\bar{x})\left(y^{*}\right) \cap\left(-\operatorname{bd} N_{C}(\bar{x})\right)=\emptyset,
$$

where "bd" refers to the topological boundary. Hence, reducing Lipschitzian stability to upper Lipschitzian stability corresponds to a transition from certain geometric objects to their boundaries. This fact becomes most evident for the simple case of one single inequality $g(x)+y \leq 0$ (i.e., $D=\mathbb{R}_{-}$): if $g$ (as a function) and $C$ (as a set) are regular in the sense of Clarke, then calmness of $M$ holds true at some point 
$(0, \bar{x})$ with $g(\bar{x})=0$, provided that bd $\partial g(\bar{x}) \cap\left(-\operatorname{bd} N_{C}(\bar{x})\right)=\emptyset$ (see Theorem 4.2 in [7]). Here, " $\partial "$ refers to either Mordukhovich's or Clarke's subdifferential (which coincide due to regularity). This last constraint qualification can be opposed again to the corresponding criterion of the Aubin property, which now takes the form $\partial g(\bar{x}) \cap$ $\left(-N_{C}(\bar{x})\right)=\emptyset$. For absent abstract constraints $\left(C=\mathbb{R}^{p}\right)$ the calmness condition reduces to $0 \notin \operatorname{bd} \partial g(\bar{x})$ (as opposed to the condition $0 \notin \partial g(\bar{x})$, which ensures the stronger Aubin property).

The aim of this paper is to study possible infinite-dimensional extensions of the previous results. For the first system in (1.3), a counterexample will show that, even for Clarke-regular data, the mentioned constraint qualification bd $\partial g(\bar{x}) \cap\left(-\operatorname{bd} N_{C}(\bar{x})\right)=$ $\emptyset$ no longer implies calmness in a Banach space setting. It does, however, for convex data, and in this case it can even be weakened again. This gives an improvement even for the finite-dimensional case. Therefore, the focus of the paper is on convex constraint systems.

2. Notation. Throughout this paper, $X$ will denote some Banach space, and $X^{*}$ its dual endowed with the strong topology. In these spaces, $B(\alpha, \beta)$ and $B^{*}(\alpha, \beta)$ are the closed balls around $\alpha$ with radius $\beta$, whereas $B^{0}(\alpha, \beta)$ refers to the corresponding open ball in $X$. By $i_{S}$ we denote the indicator function of a closed set $S \subseteq X$, and by epif the epigraph of some function $f: X \rightarrow \mathbb{R} \cup\{\infty\} . N(S ; x), \partial f$, and $\partial^{\infty} f$ refer to the normal cone to $S$ at some $x \in S$ and to the usual and singular subdifferentials of $f$, respectively, all in the sense of convex analysis. In contrast, $\partial^{c}$ represents Clarke's subdifferential. "bd" and "int" are the topological boundary and interior. For a multifunction $M: X \rightrightarrows Y$ between Banach spaces,

$$
\begin{aligned}
\operatorname{Gph} M & =\{(x, y) \in X \times Y \mid y \in M(x)\}, \\
\operatorname{range} M & =\{y \in Y \mid \exists x \in X, y \in M(x)\}, \\
M^{-1} & : Y \rightrightarrows X, \quad M^{-1}(y)=\{x \in X \mid y \in M(x)\}
\end{aligned}
$$

denote its graph, its range, and its inverse, respectively.

3. Convex constraint systems with a perturbed inequality. In this section, we consider constraint systems involving a fixed abstract constraint set and an inequality which is subject to perturbations. More precisely, we are interested in the calmness property (1.1) of the multifunction

$$
M(y):=\{x \in C \mid f(x) \leq y\} \quad(y \in \mathbb{R}),
$$

where $C$ is a closed, convex subset of some Banach space $X$ and $f$ is a convex, lower semicontinuous function. First, we state an auxiliary result. Recall from [2] that a set $S \subseteq X$ is compactly epi-Lipschitzian at some $x^{0} \in S$ if there exist a norm-compact set $K$ and a constant $r>0$ such that

$$
S \cap B\left(x^{0}, r\right)+B(0, t r) \subseteq S-t K \quad \forall t \in(0, r) .
$$

Lemma 3.1. For $C$ and $f$ as introduced above, the sum rule

$$
\partial\left(f+i_{C}\right)(\bar{x}) \subseteq \partial f(\bar{x})+N(C ; \bar{x})
$$

applies if the following constraint qualification is satisfied:

$$
\left.\begin{array}{l}
\partial^{\infty} f(\bar{x}) \cap-N(C ; \bar{x})=\{0\} \text { and } \\
C \text { or epi } f \text { is compactly epi-Lipschitzian at } \bar{x} .
\end{array}\right\}
$$


Proof. Define two closed and convex subsets of $X \times \mathbb{R}$ by $D_{1}=$ epi $f$ and $D_{2}=$ $C \times \mathbb{R}$. The first part of $\left(\mathrm{CQ}^{*}\right)$ implies that

$$
N\left(D_{1} ;(\bar{x} ; f(\bar{x}))\right) \cap-N\left(D_{2} ;(\bar{x} ; f(\bar{x}))\right)=\{0\} .
$$

Along with the second part of $\left(\mathrm{CQ}^{*}\right)$, this last relation is sufficient for the intersection rule

$$
N\left(D_{1} \cap D_{2} ;(\bar{x} ; f(\bar{x}))\right) \subseteq N\left(D_{1} ;(\bar{x} ; f(\bar{x}))\right)+N\left(D_{2} ;(\bar{x} ; f(\bar{x}))\right)
$$

(see [11, Corollary 4.5]). Now, let $x^{*} \in \partial\left(f+i_{C}\right)(\bar{x})$ be arbitrarily given, i.e., $\left\langle x^{*}, x-\right.$ $\bar{x}\rangle \leq f(x)-f(\bar{x})$ for all $x \in C$. Consequently,

$$
\left\langle\left(x^{*},-1\right),(x, t)-(\bar{x}, f(\bar{x}))\right\rangle \leq 0 \quad \forall x \in C, \forall t \geq f(x) .
$$

In other words, $\left(x^{*},-1\right) \in N\left(D_{1} \cap D_{2} ;(\bar{x} ; f(\bar{x}))\right)$, and the above intersection rule ensures that $\left(x^{*},-1\right)=\left(y^{*}, r\right)+\left(z^{*}, t\right)$ for certain $\left(y^{*}, r\right) \in N\left(D_{1} ;(\bar{x} ; f(\bar{x}))\right)$ and $\left(z^{*}, t\right) \in N\left(D_{2} ;(\bar{x} ; f(\bar{x}))\right)$. By definition of $D_{2}$, one gets $t=0$ and $z^{*} \in N(C ; \bar{x})$. It results that $r=-1$; hence $y^{*} \in \partial f(\bar{x})$ by definition of $D_{1}$. Summarizing, $x^{*} \in$ $\partial f(\bar{x})+N(C ; \bar{x})$, as we wanted to show.

Remark 3.2. The constraint qualification $\left(\mathrm{CQ}^{*}\right)$ in Lemma 3.1 is always satisfied if the convex function $f$ is continuous at $\bar{x}$ or if $\bar{x}$ is an interior point of $C$. The second part of $\left(\mathrm{CQ}^{*}\right)$ holds true whenever $X$ is finite-dimensional or the convex set $C$ has nonempty interior.

THEOREM 3.3. With the setting introduced above, the multifunction $M$ in (3.1) is calm at a point $(0, \bar{x}) \in \mathrm{Gph} M$ of its graph if one of the following conditions is satisfied:

$$
\begin{aligned}
f(\bar{x}) & <0, \\
\operatorname{bd} \partial f(\bar{x}) \cap-\operatorname{bd} N(C ; \bar{x}) & \neq \partial f(\bar{x}) \cap-N(C ; \bar{x}), \\
\operatorname{bd} \partial f(\bar{x}) \cap-\operatorname{bd} N(C ; \bar{x}) & =\emptyset, \quad \text { and } \quad\left(\mathrm{CQ}^{*}\right) .
\end{aligned}
$$

Proof. From $(0, \bar{x}) \in \mathrm{Gph} M$ it follows that $\bar{x} \in C$ and $f(\bar{x}) \leq 0$. In case of (3.2), it follows that

$$
0 \in \operatorname{int}[f(\bar{x}), \infty) \subseteq \operatorname{int~range~} M^{-1} .
$$

Since $M$ has a closed and convex graph, this last relation implies the metric regularity of $M^{-1}$ at $(\bar{x}, 0)$ by the Robinson-Ursescu theorem (see [21], [25]). However, the metric regularity of $M^{-1}$ at $(\bar{x}, 0)$ is equivalent to $M$ having the Aubin property at $(0, \bar{x})$ (cf. [3], [20], [24]), which in turn implies the calmness of $M$ at $(0, \bar{x})$. Hence, in what follows we assume that $f(\bar{x})=0$. Suppose next that (3.3) is satisfied. Then, since both $\partial f(\bar{x})$ and $-N(C ; \bar{x})$ are (strongly) closed in $X^{*}$, it holds that

$$
\operatorname{int} \partial f(\bar{x}) \cap-N(C ; \bar{x}) \neq \emptyset \quad \text { or } \quad \partial f(\bar{x}) \cap-\operatorname{int} N(C ; \bar{x}) \neq \emptyset .
$$

If the first condition of (3.6) holds, then choose $x^{*} \in \operatorname{int} \partial f(\bar{x}) \cap-N(C ; \bar{x})$. Accordingly, there exists some $\alpha>0$ such that $B^{*}\left(x^{*} ; \alpha\right) \subseteq \partial f(\bar{x})$. In other words,

$$
\left\langle x^{*}+\alpha p^{*}, x-\bar{x}\right\rangle \leq f(x)-f(\bar{x})=f(x) \quad \forall p^{*} \in B^{*}(0 ; 1), \forall x \in X .
$$

It follows that

$$
\left\langle p^{*}, x-\bar{x}\right\rangle \leq \alpha^{-1}\left(f(x)-\left\langle x^{*}, x-\bar{x}\right\rangle\right) \leq \alpha^{-1} f(x) \quad \forall p^{*} \in B^{*}(0 ; 1), \forall x \in C,
$$


since $x^{*} \in-N(C ; \bar{x})$. Consequently,

$$
\|x-\bar{x}\| \leq \alpha^{-1} f(x) \quad \forall x \in C \quad \text { and } \quad f(x) \geq 0 \quad \forall x \in C,
$$

and thus the desired calmness property of $M$ follows (with $\mathcal{U}:=X$ and $\mathcal{V}:=\mathbb{R}$ in $(1.1))$ :

$$
d(x, M(0)) \leq\|x-\bar{x}\| \leq \alpha^{-1} y=\alpha^{-1} d(y, 0) \quad \forall y \in \mathbb{R}, \forall x \in M(y) .
$$

If the second condition of (3.6) holds true, then choose $x^{*} \in \partial f(\bar{x}) \cap-\operatorname{int} N(C ; \bar{x})$. Now, there is some $\alpha>0$ such that $B^{*}\left(x^{*} ; \alpha\right) \subseteq-N(C ; \bar{x})$; hence

$$
\left\langle x^{*}-\alpha p^{*}, x-\bar{x}\right\rangle \geq 0 \quad \text { or } \quad\left\langle p^{*}, x-\bar{x}\right\rangle \leq \alpha^{-1}\left\langle x^{*}, x-\bar{x}\right\rangle \quad \forall p^{*} \in B^{*}(0 ; 1), \forall x \in C .
$$

Due to $x^{*} \in \partial f(\bar{x})$, this yields $\|x-\bar{x}\| \leq \alpha^{-1}\left\langle x^{*}, x-\bar{x}\right\rangle \leq \alpha^{-1} f(x)$ for all $x \in C$. In this way, we end up once more at relation (3.7) and, hence, at the calmness of $M$ at $(0, \bar{x})$, as above.

Finally, assume that (3.4) holds. If $0 \in \operatorname{int} \partial f(\bar{x})$, then-because of $0 \in \partial f(\bar{x}) \cap$ $-N(C ; \bar{x})-(3.3)$ is satisfied and calmness of $M$ follows as shown before. Suppose that $0 \in \operatorname{bd} \partial f(\bar{x})$. When $N(C ; \bar{x})=X^{*}$, calmness of $M$ follows again from (3.3). In the opposite case, $N(C ; \bar{x}) \neq X^{*}$, it always holds that $0 \in-\operatorname{bd} N(C ; \bar{x})$, which gives a contradiction to (3.4). It remains to check the case of

$$
0 \notin \partial f(\bar{x}) .
$$

Then, one has

$$
\partial f(\bar{x}) \cap-N(C ; \bar{x})=\emptyset \quad \text { or } \quad \partial f(\bar{x}) \subseteq-\operatorname{int} N(C ; \bar{x}) .
$$

To verify this statement, assume that neither of the two conditions is satisfied. Then, there exist $x_{1}^{*}, x_{2}^{*} \in \partial f(\bar{x})$ such that $x_{1}^{*} \in-N(C ; \bar{x})$ and $x_{2}^{*} \notin-\operatorname{int} N(C ; \bar{x})$. The convexity of $\partial f(\bar{x})$ and $-N(C ; \bar{x})$ guarantees the existence of some $x^{*}$ (on the line segment $\left.\left[x_{1}^{*}, x_{2}^{*}\right]\right)$ such that $x^{*} \in \partial f(\bar{x}) \cap-\mathrm{bd} N(C ; \bar{x})$. By the cone property of $N(C ; \bar{x})$, one has that $t x^{*} \in-\mathrm{bd} N(C ; \bar{x})$ for all $t>0$. Due to the closedness of $\partial f(\bar{x})$, there must be some $t^{*}>0$ such that $t^{*} x^{*} \notin \partial f(\bar{x})$ (otherwise we have a contradiction with (3.8)). But then, since $x^{*} \in \partial f(\bar{x})$, there must exist some $\hat{t}>0$ such that $\hat{t} x^{*} \in \operatorname{bd} \partial f(\bar{x})$. At the same time, $\hat{t} x^{*} \in-\operatorname{bd} N(C ; \bar{x})$, whence a contradiction to (3.4), and (3.9) must hold true.

Now, the first case of (3.9) implies the existence of some $x^{\prime} \in C$ such that $f\left(x^{\prime}\right)<0$ (Slater's condition). Indeed, negating Slater's condition means that $\bar{x}$ is a minimum of $f$ over $C$ or, equivalently, a free minimum of the lower semicontinuous function $f+i_{C}$. Consequently,

$$
0 \in \partial\left(f+i_{C}\right)(\bar{x}) \subseteq \partial f(\bar{x})+N(C ; \bar{x}),
$$

where we have applied Lemma 3.1. However, the obtained relation contradicts the first case of (3.9). Hence, Slater's condition is satisfied, and one has (3.5) with $\bar{x}$ replaced by $x^{\prime}$. Consequently, the calmness of $M$ at $\bar{x}$ follows as in the lines below (3.5).

Concerning the second case of (3.9), assume first that $\partial f(\bar{x})=\emptyset$. Then, we are back to the first case of (3.9) already considered. Finally, if $\partial f(\bar{x}) \neq \emptyset$, then the second case of (3.9), along with (3.4), yields (3.3), and the calmness of $M$ at $(0, \bar{x})$ follows again. 
For missing abstract constraints, a much simpler characterization of calmness can be derived from Theorem 3.3, as follows.

Corollary 3.4. Let $X$ be a Banach space, and $f: X \rightarrow \mathbb{R} \cup\{\infty\}$ a convex, lower semicontinuous function. Then, the multifunction $M(y):=f^{-1}(-\infty, y]$ is calm at a point $(0, \bar{x})$ with $f(\bar{x}) \leq 0$ if

$$
f(\bar{x})<0 \quad \text { or } \quad 0 \notin \operatorname{bd} \partial f(\bar{x}) .
$$

Proof. The first condition of (3.10) coincides with (3.2); thus it suffices to consider the second condition of (3.10). Evidently, in the setting of (3.1), we have $C=X$; hence $N(C ; \bar{x})=\operatorname{bd} N(C ; \bar{x})=\{0\}$. Along with $0 \notin$ bd $\partial f(\bar{x})$, this provides

$$
\operatorname{bd} \partial f(\bar{x}) \cap-\operatorname{bd} N(C ; \bar{x})=\emptyset ;
$$

hence (3.4) is satisfied. (Note that $\left(\mathrm{CQ}^{*}\right)$ is trivially satisfied in the context of this corollary; see Remark 3.2.)

Note that in the setting of Corollary 3.4 we have the following implications:

$$
(3.3) \Longrightarrow 0 \in \operatorname{int} \partial f(\bar{x}) \Longrightarrow(3.4) \Longrightarrow(3.10) \text {. }
$$

Hence, in contrast to the alternative of conditions (3.3) and (3.4) in Theorem 3.3, there is no point in considering (3.3) here in addition to (3.10). In the general setting of Theorem 3.3, however, it is no longer true that (3.3) implies (3.4), as can be seen from the second part of Example 3.6 below.

Remark 3.5. For finite-dimensional $X$, condition (3.4) - with the convex subdifferential replaced by Clarke - was shown in [7] to be sufficient for calmness of the multifunction $M$ if $f$ is locally Lipschitzian and both $f$ and $C$ are regular in the sense of Clarke. Theorem 3.3 demonstrates that this condition can be weakened to "(3.3) or (3.4)" in the convex case even if $X$ is infinite-dimensional. More precisely, one has the following structure of constraint qualifications here (assuming that $f$ is continuous at $\bar{x} \in C$ and $f(\bar{x})=0)$ :

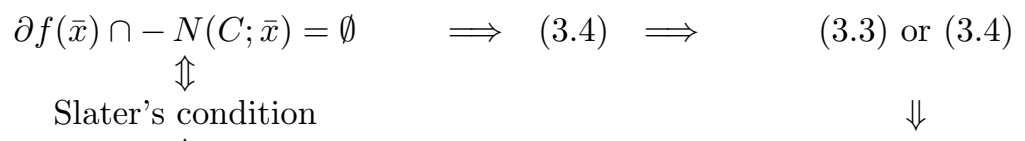

Aubin property of $M$ at $(0, \bar{x}) \quad$ calmness of $M$ at $(0, \bar{x})$.

In this diagram, we mean by Slater's condition the existence of some $x^{*} \in C$ such that $f\left(x^{*}\right)<0$ (which is equivalent to the Aubin property of $M$ at $(0, \bar{x})$ or to the metric regularity of $M^{-1}$ at $(\bar{x}, 0)$ by the Robinson-Ursescu theorem).

We continue with some examples.

EXAMPLE 3.6. The three constraint qualifications considered in Remark 3.5 are strictly different. Setting, for instance, $f(x)=|x|, C=\mathbb{R}, \bar{x}=0$, Slater's condition is obviously violated (and also $0 \in \partial f(\bar{x}) \cap-N(C ; \bar{x}) \neq \emptyset$ ), whereas (3.4) holds true:

$$
\operatorname{bd} \partial f(\bar{x}) \cap-\operatorname{bd} N(C ; \bar{x})=\{-1,1\} \cap\{0\}=\emptyset .
$$

Indeed, $M$ is calm at $(0, \bar{x})$ but fails to have the Aubin property there. Another example is $f(x)=f\left(x_{1}, x_{2}\right)=\|x\|, C=\left\{\left(x_{1}, x_{2}\right) \mid x_{1} \geq 0\right\}$. Then, at $\bar{x}=(0,0)$, one has

$$
\begin{aligned}
\operatorname{bd} \partial f(\bar{x}) \cap-\operatorname{bd} N(C ; \bar{x}) & =\left\{\left(x_{1}, x_{2}\right) \mid x_{1}^{2}+x_{2}^{2}=1, x_{1} \geq 0, x_{2}=0\right\}=\{(1,0)\}, \\
\partial f(\bar{x}) \cap-N(C ; \bar{x}) & =\left\{\left(x_{1}, x_{2}\right) \mid x_{1}^{2}+x_{2}^{2} \leq 1, x_{1} \geq 0, x_{2}=0\right\} \\
& =\operatorname{conv}\{(0,0),(1,0)\} .
\end{aligned}
$$


Hence, (3.4) is violated here, whereas (3.3) is satisfied, and thus Theorem 3.3 ensures the calmness of $M$ at $(0, \bar{x})$. Again, $M$ fails to have the Aubin property.

The following example demonstrates that Theorem 3.3 provides a sufficient but not a necessary condition for the calmness of the multifunction $M$ considered there.

EXAmple 3.7. Let $X=C=\mathbb{R}, \bar{x}=0$, and $f(x)=\max \{x, 0\}$. Then, $(0, \bar{x}) \in$ $\operatorname{Gph} M, f(\bar{x})=0$, and $M(0)=\mathbb{R}_{-}$. One has $M(y)=\emptyset$ for $y<0$, and $M(y)=$ $(-\infty, y]$ for $y \geq 0$; hence $d(x, M(0)) \leq d(y, 0)$ for all $y \in \mathbb{R}$ and all $x \in M(y)$. This means calmness of $M$ at $(0, \bar{x})$. On the other hand, since $\partial f(\bar{x})=[0,1],(3.10)$ is violated, which implies the violation of both (3.4) and (3.3).

Note that, in the last example, $M$ was a polyhedral multifunction; hence it seems that one cannot recover by Theorem 3.3 Robinson's result mentioned in the introduction. However, this will be possible after some modification, following the ideas of [12].

The next example requires some technical work. It illustrates the limitation of Theorem 3.3 to convex data. In finite dimensions, the condition " $f(\bar{x})<0$ or $0 \notin \operatorname{bd} \partial^{c} f(\bar{x})$ " (i.e., (3.10) with the convex subdifferential replaced by Clarke's) was found in [7] to ensure calmness of the multifunctions (3.1) without abstract constraints (i.e., $C=X$ ) as long as $f$ is regular at $\bar{x}$ in the sense of Clarke. This is no longer true in infinite dimensions unless the data are restricted to be convex as in Corollary 3.4.

EXAMPLE 3.8. For $k \in \mathbb{N}$, let $\tau_{k} \in\left(0, k^{-2}\right)$ be the unique solution of $\tau+k \sqrt{\tau}=1$. Define the sequence of real functions

$$
\varphi_{k}(\tau):= \begin{cases}|\tau|(1-k \sqrt{|\tau|}) & \text { if } \tau \in\left[-\tau_{k}, \tau_{k}\right] \\ \tau_{k}^{2} & \text { if }|\tau| \geq \tau_{k}\end{cases}
$$

Elementary analysis shows that each $\varphi_{k}$ is (globally) Lipschitz continuous with modulus 1 and regular at zero in the sense of Clarke. (Close to the origin, each $\varphi_{k}$ can be represented as the maximum of two $\mathcal{C}^{1}$ - functions.) Furthermore,

$$
\varphi_{k} \geq 0, \quad \varphi_{k}(\tau)=0 \Longleftrightarrow \tau=0, \quad \text { and } \quad \varphi_{k}\left(\tau_{k}\right)=\tau_{k}^{2} \quad \forall k \in \mathbb{N}, \forall \tau \in \mathbb{R} .
$$

Now, let $X=l^{1}$, and define $f: X \rightarrow \mathbb{R}$ by $f(x):=\sum_{k=1}^{\infty} \varphi_{k}\left(x_{k}\right)$. Evidently, $f(0)=0$ by (3.12). Since $\varphi_{k}(\tau) \leq \tau_{k}^{2} \leq k^{-4}$ for all $\tau \in \mathbb{R}$ and all $k \in \mathbb{N}, f$ is well defined. For arbitrary $x, y \in X$, one has

$$
\begin{aligned}
|f(x)-f(y)| & =\left|\sum_{k=1}^{\infty}\left(\varphi_{k}\left(x_{k}\right)-\varphi_{k}\left(y_{k}\right)\right)\right| \leq \sum_{k=1}^{\infty}\left|\varphi_{k}\left(x_{k}\right)-\varphi_{k}\left(y_{k}\right)\right| \\
& \leq \sum_{k=1}^{\infty}\left|x_{k}-y_{k}\right|=\|x-y\|_{1}
\end{aligned}
$$

hence $f$ is (globally) Lipschitz continuous with modulus 1.

Next, we calculate Clarke's directional derivative $f^{0}(0 ; h)$ of $f$ at zero in arbitrary direction $h \in X$. By definition (see [4]), one has

$$
\begin{aligned}
f^{0}(0 ; h) & =\limsup _{t \downarrow 0, x \rightarrow 0} \frac{f(x+t h)-f(x)}{t}=\lim _{n \rightarrow \infty} \frac{f\left(x^{(n)}+t^{(n)} h\right)-f\left(x^{(n)}\right)}{t^{(n)}} \\
& =\lim _{n \rightarrow \infty} \sum_{k=1}^{\infty} \frac{\varphi_{k}\left(x_{k}^{(n)}+t^{(n)} h_{k}\right)-\varphi_{k}\left(x_{k}^{(n)}\right)}{t^{(n)}},
\end{aligned}
$$


where $x^{(n)} \rightarrow 0$ and $t^{(n)} \downarrow 0$ are suitable sequences realizing the above limsup as a limit. Now, we fix an arbitrary $k^{\prime} \in \mathbb{N}$. Assume that there exist $\varepsilon>0$ and $n_{0} \in \mathbb{N}$ such that

$$
\frac{\varphi_{k^{\prime}}\left(x_{k^{\prime}}^{(n)}+t^{(n)} h_{k^{\prime}}\right)-\varphi_{k^{\prime}}\left(x_{k^{\prime}}^{(n)}\right)}{t^{(n)}} \leq \frac{\varphi_{k^{\prime}}\left(t^{(n)} h_{k^{\prime}}\right)}{t^{(n)}}-\varepsilon \quad \forall n \geq n_{0}
$$

In order to lead (3.14) to a contradiction, define a sequence $\tilde{x}^{(n)} \in X$ by

$$
\tilde{x}_{k}^{(n)}:=\left\{\begin{array}{ll}
x_{k}^{(n)}, & k \neq k^{\prime}, \\
0, & k=k^{\prime}
\end{array} \quad \forall k, n \in \mathbb{N}\right.
$$

It follows that $\tilde{x}^{(n)} \rightarrow 0$ and, in view of $(3.12)$,

$$
\begin{aligned}
\frac{f\left(\tilde{x}^{(n)}+t^{(n)} h\right)-f\left(\tilde{x}^{(n)}\right)}{t^{(n)}} & =\sum_{k=1, k \neq k^{\prime}}^{\infty} \frac{\varphi_{k}\left(x_{k}^{(n)}+t^{(n)} h_{k}\right)-\varphi_{k}\left(x_{k}^{(n)}\right)}{t^{(n)}}+\frac{\varphi_{k^{\prime}}\left(t^{(n)} h_{k^{\prime}}\right)}{t^{(n)}} \\
& \geq \sum_{k=1}^{\infty} \frac{\varphi_{k}\left(x_{k}^{(n)}+t^{(n)} h_{k}\right)-\varphi_{k}\left(x_{k}^{(n)}\right)}{t^{(n)}}+\varepsilon \\
& =\frac{f\left(x^{(n)}+t^{(n)} h\right)-f\left(x^{(n)}\right)}{t^{(n)}}+\varepsilon
\end{aligned}
$$

for $n \geq n_{0}$, whence the contradiction with (3.13),

$$
\limsup _{t \downarrow 0, x \rightarrow 0} \frac{f(x+t h)-f(x)}{t} \geq f^{0}(0 ; h)+\varepsilon .
$$

Therefore, we may negate (3.14) in order to obtain a subsequence symbolized by the index $m(n)$ such that

$$
\begin{aligned}
\liminf _{n \rightarrow \infty} & \frac{\varphi_{k^{\prime}}\left(x_{k^{\prime}}^{(m(n))}+t^{(m(n))} h_{k^{\prime}}\right)-\varphi_{k^{\prime}}\left(x_{k^{\prime}}^{(m(n))}\right)}{t^{(m(n))}} \\
& \geq \lim _{n \rightarrow \infty} \frac{\varphi_{k^{\prime}}\left(t^{(m(n))} h_{k^{\prime}}\right)}{t^{(m(n))}}=d \varphi_{k^{\prime}}\left(0 ; h_{k^{\prime}}\right)=\varphi_{k^{\prime}}^{0}\left(0 ; h_{k^{\prime}}\right) \\
& \geq \limsup _{n \rightarrow \infty} \frac{\varphi_{k^{\prime}}\left(x_{k^{\prime}}^{(m(n))}+t^{(m(n))} h_{k^{\prime}}\right)-\varphi_{k^{\prime}}\left(x_{k^{\prime}}^{(m(n))}\right)}{t^{(m(n))}},
\end{aligned}
$$

where " $d \varphi_{k^{\prime}}$ " refers to the usual directional derivative, which, by the already stated regularity of $\varphi_{k^{\prime}}$ in the sense of Clarke, exists and coincides with $\varphi_{k^{\prime}}^{0}$. From the definition of $\varphi_{k^{\prime}}$, one calculates $d \varphi_{k^{\prime}}\left(0 ; h_{k^{\prime}}\right)=\left|h_{k^{\prime}}\right|$. Since $k^{\prime}$ was arbitrarily fixed, (3.15) provides

$$
\lim _{n \rightarrow \infty} \frac{\varphi_{k}\left(x_{k}^{(m(n))}+t^{(m(n))} h_{k}\right)-\varphi_{k}\left(x_{k}^{(m(n))}\right)}{t^{(m(n))}}=\left|h_{k}\right| \quad \forall k \in \mathbb{N} .
$$

This finally allows us to interchange limit and summation in the last term of (3.13) (upon passing to the subsequence $m(n)$ there too):

$$
f^{0}(0 ; h)=\sum_{k=1}^{\infty}\left|h_{k}\right|=\|h\|_{1} \quad \forall h \in X
$$


Consequently, $\partial^{c} f(0)=B_{1}$, where $\partial^{c}$ denotes Clarke's subdifferential and $B_{1}$ is the unit ball in $X$.

Next, we verify that $f$ is regular at 0 in the sense of Clarke. To this aim, we calculate its usual directional derivative at 0 in arbitrary direction $h$. Since for each sequence $t^{(n)} \downarrow 0$ it holds that

$$
\lim _{n \rightarrow \infty} \frac{\varphi_{k}\left(t^{(n)} \tilde{h}\right)}{t^{(n)}}=d \varphi_{k}(0 ; \tilde{h})=|\tilde{h}| \quad \forall \tilde{h} \in \mathbb{R}, \forall k \in \mathbb{N},
$$

one may interchange limit and summation once more:

$$
\|h\|_{1}=\sum_{k=1}^{\infty} \lim _{n \rightarrow \infty} \frac{\varphi_{k}\left(t^{(n)} h_{k}\right)}{t^{(n)}}=\lim _{n \rightarrow \infty} \sum_{k=1}^{\infty} \frac{\varphi_{k}\left(t^{(n)} h_{k}\right)}{t^{(n)}}=\lim _{n \rightarrow \infty} \frac{f\left(t^{(n)} h\right)-f(0)}{t^{(n)}} .
$$

As $t^{(n)} \downarrow 0$ was arbitrary, it follows that $d f(0 ; h)=\|h\|_{1}=f^{0}(0 ; h)$; hence $f$ is regular in the sense of Clarke.

Finally, we consider the multivalued mapping $M: \mathbb{R} \rightrightarrows X$ defined by $M(t):=$ $\{x \in X \mid f(x) \leq t\}$. This is exactly the setting of (3.1) with abstract constraints missing $(X=C)$. By the definition of $f$ and (3.12), one has

$$
f(x) \geq 0 \quad \forall x \in X \quad \text { and } \quad f(x)=0 \Longleftrightarrow x=0 .
$$

Hence, $M(0)=\{0\}$. Define a sequence $z^{(n)}=\left(0, \ldots, 0, \tau_{n}, 0,0, \ldots\right) \in X$, with $\tau_{n}$ at position $n$. Then, again by (3.12),

$$
d\left(z^{(n)}, M(0)\right)=\left\|z^{(n)}\right\|_{1}=\tau_{n} \quad \text { and } \quad f\left(z^{(n)}\right)=\varphi_{n}\left(\tau_{n}\right)=\tau_{n}^{2} \quad \forall n \in \mathbb{N} .
$$

Setting $y^{(n)}:=f\left(z^{(n)}\right)$, we have constructed sequences $z^{(n)}, y^{(n)}$ such that $z^{(n)} \in$ $M\left(y^{(n)}\right), z^{(n)} \rightarrow 0, y^{(n)} \rightarrow 0$ (because of $\tau_{n} \in\left(0, n^{-2}\right)$ ). From here, we derive that $M$ fails to be calm at $(0,0)$ :

$$
d\left(z^{(n)}, M(0)\right)=\tau_{n}^{-1} f\left(z^{(n)}\right)=\tau_{n}^{-1} d\left(f\left(z^{(n)}\right), 0\right) \geq n^{2} d\left(f\left(z^{(n)}\right), 0\right)
$$

(again by $\tau_{n} \in\left(0, n^{-2}\right)$ ), which contradicts (1.1). On the other hand, we have seen that $\partial^{c} f(0)=B_{1}$; hence $0 \in \operatorname{int} \partial^{c} f(0)$, and the constraint qualification " $f(\bar{x})<0$ or $0 \notin \operatorname{bd} \partial^{c} f(\bar{x}) "$ - which was sufficient for calmness in the regular, finite-dimensional and in the convex, infinite-dimensional cases - is evidently satisfied. However, the same constraint qualification (to which the conditions (3.4) and (3.3) reduce when $C=X$ ) does not imply calmness in the regular, infinite-dimensional case, as was shown in this example.

The next result is an immediate application of Theorem 3.3 to the characterization of calmness for nonstructured multifunctions.

Corollary 3.9. Let $X$ be a Banach space, $Y$ a metric space, $M: X \rightrightarrows Y$ a multifunction with closed values, and $(\bar{x}, \bar{y}) \in \mathrm{Gph} M$. Assume further that

(1) the distance function $d(\bar{y}, M(\cdot))$ is convex and lower semicontinuous in a neighborhood of $\bar{x}$;

(2) $0 \notin \operatorname{bd} \partial d(\bar{y}, M(\cdot))(\bar{x})$.

Then $M^{-1}$ is calm at $(\bar{y}, \bar{x})$.

Proof. Corollary 3.4 immediately provides calmness at $(\bar{y}, \bar{x})$ of the multifunction $P: \mathbb{R} \rightrightarrows X$ defined by

$$
P(t):=\{x \in X \mid d(\bar{y}, M(x)) \leq t\} .
$$


This means the existence of some $L>0, \varepsilon>0$ such that

$$
d(x, P(0)) \leq L|t| \quad \forall t \in(-\varepsilon, \varepsilon), \forall x \in B^{0}(\bar{x} ; \varepsilon) \cap P(t) .
$$

Since $P(0)=M^{-1}(\bar{y})$ and $M^{-1}(y) \subseteq P(d(\bar{y}, y))$ for all $y \in Y$, the calmness of $M^{-1}$ at $(\bar{y}, \bar{x})$ follows:

$$
d\left(x, M^{-1}(\bar{y})\right)=d(x, P(0)) \leq L d(\bar{y}, y) \quad \forall y \in B^{0}(\bar{y} ; \varepsilon), \forall x \in B^{0}(\bar{x} ; \varepsilon) \cap M^{-1}(y) .
$$

Note that Corollary 3.9(2) is far removed from being necessary for calmness or even the stronger Aubin property.

Example 3.10. Consider $M(x):=[x, \infty)$ at $(0,0) \in \operatorname{Gph} M$. Since $d(0, M(x))=$ $\max \{0, x\}$, Corollary 3.9(1) is satisfied, whereas condition (2) is violated. On the other hand, the inverse multifunction $M^{-1}(y)=\{x \mid x \leq y\}$ is easily seen to satisfy the Aubin property (1.2) and, hence, calmness at $(0,0)$.

At the end of this section we want briefly to compare our conditions for the calmness of system (3.1) with similar conditions which were obtained in the context of error bounds. First, recall that the calmness of (3.1) is equivalent to the existence of a local error bound. A rigorous comparison is difficult because the obtained conditions may differ by many features (e.g., local vs. global error bounds, primal vs. dual conditions, finite- vs. infinite-dimensional spaces, point vs. neighborhood conditions). However, one could at least try to reduce all these conditions to a simple common setting, where $C=X$ is finite-dimensional and $f$ is convex and finite-valued. As far as dual conditions for error bounds are concerned, they usually come down to just Slater's condition in dual form, " $0 \notin \partial f(\bar{x})$ " in that situation (see, e.g., [14, condition (ACQ11)], [15, Section 3, Corollary 2(b)], or [5, Theorem 1]). Slater's condition, however, is much stronger than our condition (3.10), as was shown in Example 3.6 (see also (3.11)). A primal condition for calmness proposed in [17, Theorem 13] is

$$
0 \in \operatorname{int}\left(f(C)+\mathbb{R}_{+}\right) .
$$

However, in our setting, with $C=X$, this relation obviously reduces to Slater's condition in primal form: " $\exists x^{*}: f\left(x^{*}\right)<0$." Hence, the same remarks as above apply with respect to condition (3.10). A mixed primal/dual condition was derived in [15, Theorem 1] for finite dimensions:

$$
\exists \gamma>0: f^{\prime}(\bar{x} ; d) \geq \gamma^{-1}\|d\| \quad \forall \bar{x} \in f^{-1}(0), \forall d \in N\left(f^{-1}(-\infty, 0] ; \bar{x}\right) .
$$

Here, $f^{\prime}$ refers to the directional derivative of $f$. It is elementary to verify that in the special setting considered here $(C=X),(3.10)$ implies (3.16). In particular, (3.16) could be applied in Example 3.7, where (3.10) failed. On the other hand, (3.16) is not a point condition by relying on the whole solution set $f^{-1}(-\infty, 0]$. This could make its verification in general problems less convenient than that of (3.10), which is sufficient at least for local error bounds. A similar comparison holds true for a nonsmooth Abadie's constraint qualification formulated in [19].

4. Calmness of the intersection of two sets. In this section, we turn to the calmness property with respect to two sets. To this aim, let $C, D \subseteq X$ be closed, convex subsets such that $\bar{x} \in C \cap D$. We want to characterize the calmness of the multivalued mapping $Q: \mathbb{R} \rightrightarrows X$ defined by

$$
Q(t):=\{x \in X \mid d(x, C)+d(x, D) \leq t\}
$$


at the point $(0, \bar{x}) \in \operatorname{Gph} Q$.

Lemma 4.1. $Q$ is calm at $(0, \bar{x}) \in \operatorname{Gph} Q$, provided that

$$
\operatorname{int} N(D ; \bar{x}) \cap-N(C ; \bar{x}) \neq \emptyset \text {. }
$$

Proof. Choose $x^{*} \in \operatorname{int} N(D ; \bar{x}) \cap-N(C ; \bar{x})$. From $x^{*} \in \operatorname{int} N(D ; \bar{x})$, it follows, similarly to the proof of Theorem 3.3, that there exists some $\alpha>0$ such that

$$
\alpha\|x-\bar{x}\|+\left\langle x^{*}, x-\bar{x}\right\rangle \leq 0 \quad \forall x \in D .
$$

Hence, $\bar{x}$ is a minimizer of the function $\left\langle x^{*}, \bar{x}-\cdot\right\rangle-\alpha\|\cdot-\bar{x}\|$ on the set $D$. Now, using a well-known penalization argument, which appeals to the Lipschitz constant of the function involved, it follows that there exists some $\varepsilon>0$ such that

$$
\left\langle x^{*}, \bar{x}-x\right\rangle-\alpha\|x-\bar{x}\|+\left(\left\|x^{*}\right\|+\alpha\right) d(x, D) \geq 0 \quad \forall x \in B(\bar{x} ; \varepsilon),
$$

whence, by $x^{*} \in-N(C ; \bar{x})$,

$$
-\alpha\|x-\bar{x}\|+\left(\left\|x^{*}\right\|+\alpha\right) d(x, D) \geq 0 \quad \forall x \in B(\bar{x} ; \varepsilon) \cap C .
$$

In other words, $\bar{x}$ is a local minimizer of the function $-\alpha\|\cdot-\bar{x}\|+\left(\left\|x^{*}\right\|+\alpha\right) d(\cdot, D)$ on the set $C$. Now, upon repeating the same penalization argument, one arrives at

$$
-\alpha\|x-\bar{x}\|+\left(\left\|x^{*}\right\|+\alpha\right) d(x, D)+\left(\left\|x^{*}\right\|+2 \alpha\right) d(x, C) \geq 0 \quad \forall x \in B\left(\bar{x} ; \varepsilon^{\prime}\right)
$$

for some $\varepsilon^{\prime}>0$. This, however, is the desired calmness property

$$
d(x, Q(0)) \leq\|x-\bar{x}\| \leq \alpha^{-1}\left(\left\|x^{*}\right\|+2 \alpha\right)(d(x, D)+d(x, C)) \leq \alpha^{-1}\left(\left\|x^{*}\right\|+2 \alpha\right)|t|,
$$

which holds true for all $t \in \mathbb{R}$ and all $x \in B\left(\bar{x} ; \varepsilon^{\prime}\right) \cap Q(t)$.

Next, we need an auxiliary result, which is of independent interest.

Lemma 4.2. If one of the sets $C$ or $D$ is compactly epi-Lipschitzian in a neighborhood of $\bar{x}$, then

$$
N(D ; \bar{x}) \cap-N(C ; \bar{x})=\{0\} \Longleftrightarrow 0 \in \operatorname{int}(D-C \cap B(\bar{x}, 1)) .
$$

Proof. $(\Longrightarrow)$ For symmetry reasons, one may take, e.g., $D$ to be compactly epiLipschitzian in a neighborhood of $\bar{x}$. Assume that

$$
0 \notin \operatorname{int}(D-C \cap B(\bar{x}, 1))=\operatorname{int} \overline{(D-C \cap B(\bar{x}, 1))}
$$

(the equality follows from [22, Lemma 1]). Accordingly, there exists a sequence $b_{n} \rightarrow 0$ with

$$
b_{n} \notin \overline{D-C \cap B(\bar{x}, 1))} .
$$

The separation theorem provides a corresponding sequence $x_{n}^{*} \in X^{*}$ such that $\left\|x_{n}^{*}\right\|=$ 1 and

$$
\left\langle x_{n}^{*}, b_{n}\right\rangle \leq\left\langle x_{n}^{*}, d-\bar{x}\right\rangle \quad \forall d \in D, \quad\left\langle x_{n}^{*}, b_{n}\right\rangle \leq\left\langle x_{n}^{*}, \bar{x}-c\right\rangle \quad \forall c \in C \cap B(\bar{x}, 1) .
$$

The first relation of (4.2) yields that $\left\langle x_{n}^{*}, \bar{x}\right\rangle \leq \inf _{d \in D}\left\langle x_{n}^{*}, d\right\rangle+\left\|b_{n}\right\|$. Now Ekeland's variational principle provides a sequence $d_{n} \in D$ such that

$$
\left\|d_{n}-\bar{x}\right\| \leq \sqrt{\left\|b_{n}\right\|} \quad \text { and } \quad\left\langle x_{n}^{*}, d_{n}\right\rangle \leq\left\langle x_{n}^{*}, d\right\rangle+\sqrt{\left\|b_{n}\right\|}\left\|d_{n}-d\right\| \quad \forall d \in D .
$$


The second relation of (4.3) entails that $-x_{n}^{*} \in N\left(D ; d_{n}\right)+B^{*}\left(0, \sqrt{\left\|b_{n}\right\|}\right)$; hence there are sequences $z_{n}^{*} \in N\left(D ; d_{n}\right)$ and $b_{n}^{*}$ with $\left\|b_{n}^{*}\right\| \leq \sqrt{\left\|b_{n}\right\|}$ such that $z_{n}^{*}+x_{n}^{*}+b_{n}^{*}=0$. In particular, $\left\|z_{n}^{*}\right\| \rightarrow 1$. Thus, the sequence $z_{n}^{*}$ is bounded and, hence, there exists a weak* convergent subnet $z_{\lambda}^{*} \rightarrow_{w^{*}} z^{*}$. Now, since $z_{\lambda}^{*} \in N\left(D ; d_{\lambda}\right)$, this last convergence, $d_{\lambda} \rightarrow \bar{x}$ (see the first relation of (4.3)), and the very definition of the normal cone to convex sets yield that $z^{*} \in N(D ; \bar{x})$. Now, the assumed property of $D$ being compactly epi-Lipschitzian in a neighborhood $\mathcal{V}_{\bar{x}}$ of $\bar{x}$ results in the inclusion

$$
N(D ; x) \subseteq\left\{x^{*} \mid\left\|x^{*}\right\| \leq \max _{i=1, \ldots, k}\left\langle x^{*}, h_{i}\right\rangle\right\} \quad \forall x \in \mathcal{V}_{\bar{x}} \cap D
$$

for certain $h_{i} \in X(i=1, \ldots, k)$. From $d_{\lambda} \rightarrow \bar{x}$, one derives that

$$
\max _{i=1, \ldots, k}\left\langle z_{\lambda}^{*}, h_{i}\right\rangle \geq\left\|z_{\lambda}^{*}\right\| \text {. }
$$

Consequently, $z^{*} \neq 0$. On the other hand, we also have that $x_{\lambda}^{*}=-z_{\lambda}^{*}-b_{\lambda}^{*}-w^{*}-z^{*}$, which together with the second part of (4.2) provides

$$
\left\langle-z^{*}, \bar{x}-c\right\rangle \leftarrow_{w^{*}}\left\langle x_{\lambda}^{*}, \bar{x}-c\right\rangle \geq\left\langle x_{\lambda}^{*}, b_{\lambda}\right\rangle \rightarrow 0 \quad \forall c \in C \cap B(\bar{x}, 1),
$$

whence $z^{*} \in-N(C ; \bar{x})$. Summarizing, there is some $z^{*} \neq 0$ with $z^{*} \in N(D ; \bar{x}) \cap$ $-N(C ; \bar{x})$. This contradicts our assumption.

$(\Longleftarrow)$ Choose an arbitrary $x^{*} \in N(D ; \bar{x}) \cap-N(C ; \bar{x})$. Then,

$$
\left\langle x^{*}, d-\bar{x}\right\rangle \leq 0 \quad \forall d \in D \quad \text { and } \quad\left\langle x^{*}, \bar{x}-c\right\rangle \leq 0 \quad \forall c \in C .
$$

In other words, $\left\langle x^{*}, d-c\right\rangle \leq 0$ for all $d \in D$ and all $c \in C$. However, since by assumption $0 \in \operatorname{int}(D-C)$, it results that $x^{*}=0$, as we wanted to show.

THEOREM 4.3. Let one of the sets $C$ or $D$ be compactly epi-Lipschitzian at $\bar{x}$. Then, $Q$ is calm at $(0, \bar{x})$ under the following condition:

$$
\operatorname{bd} N(D ; \bar{x}) \cap-\operatorname{bd} N(C ; \bar{x})=\{0\} .
$$

Proof. For the case in which $N(D ; \bar{x}) \cap-N(C ; \bar{x})=\{0\}$, Lemma 4.2 ensures that $0 \in \operatorname{int}(D-C)$. Since $D-C$ equals the range of the multifunction $M: X \rightrightarrows X$ defined by

$$
M(x)= \begin{cases}-x+D, & x \in C, \\ \emptyset, & x \notin C,\end{cases}
$$

we have $0 \in$ int range $M$, and the Robinson-Ursescu theorem yields the metric regularity of $M$ at the point $(\bar{x}, 0)$ of its graph. This property means the existence of $L, \varepsilon>0$ such that

$$
d\left(x, M^{-1}(y)\right) \leq L d(y, M(x)) \quad \forall x \in B(\bar{x}, \varepsilon), \forall y \in B(0, \varepsilon) .
$$

With $M^{-1}(y)=C \cap(D-y)$ and fixing $y:=0$, one arrives at

$$
\begin{gathered}
d(x, C \cap D) \leq L d(x, D) \quad \forall x \in B(\bar{x}, \varepsilon) \cap C \text {; hence } \\
d(x, C \cap D) \leq(L+1)(d(x, D)+d(x, C)) \quad \forall x \in B(\bar{x}, \varepsilon) .
\end{gathered}
$$

This, of course, is the calmness of the multifunction $Q$ at $(0, \bar{x})$.

Otherwise $(N(D ; \bar{x}) \cap-N(C ; \bar{x}) \neq\{0\}),(4.4)$ implies that

$$
\operatorname{int} N(D ; \bar{x}) \cap-N(C ; \bar{x}) \neq \emptyset \quad \text { or } \quad N(D ; \bar{x}) \cap-\operatorname{int} N(C ; \bar{x}) \neq \emptyset .
$$

In both cases, Lemma 4.1 yields the desired result. 
5. The differentiable nonconvex case. In this section we briefly return to the constraint system (3.1), with a convex closed subset $C \subseteq X$ as before, but with a (strictly) differentiable function $f$. Theorem 3.3 has shown that, in the completely convex case $(C$ and $f)$, each of the constraint qualifications (3.4), (3.3) is sufficient for the calmness of (3.1). On the other hand, we know by Example 3.8 that neither of the two conditions ensures calmness if $f$ is just regular in the sense of Clarke. Since, in that example, $f$ was nondifferentiable, the question arises of whether a positive result can be expected in the smooth case. The answer is affirmative even for a finite number of inequalities.

Theorem 5.1. Consider a multifunction $M: \mathbb{R}^{m} \rightrightarrows X$ defined by

$$
M(y):=\{x \in C \mid f(x) \leq y\} \quad\left(y \in \mathbb{R}^{m}\right),
$$

where $C \subseteq X$ is convex and closed and $f: X \rightarrow \mathbb{R}^{m}$ is strictly differentiable. Then, the constraint qualification

$$
\operatorname{conv}\left\{\nabla f_{i}(\bar{x})\right\}_{i \in I(\bar{x})} \cap-\operatorname{bd} N(C ; \bar{x})=\emptyset
$$

implies the calmness of $M$ at $(0, \bar{x}) \in \mathrm{Gph} M$. Here, $f_{i}$ denote the components of $f$, and $I(x)=\left\{i \in\{1, \ldots, m\} \mid f_{i}(x)=0\right\}$ refers to the set of active indices.

Proof. Assume first that $\operatorname{conv}\left\{\nabla f_{i}(\bar{x})\right\}_{i \in I(\bar{x})} \cap-N(C ; \bar{x})=\emptyset$. Then, the strict differentiability assumption on $f$ allows us to apply Theorem 2.4 in [13] in order to derive the metric regularity of $M^{-1}$ at $(\bar{x}, 0)$, which is equivalent to the Aubin property of $M$ at $(0, \bar{x})$ and, hence, implies calmness of $M$ at $(0, \bar{x})$. In the opposite case, (5.1) guarantees the existence of some $x^{*} \in \operatorname{conv}\left\{\nabla f_{i}(\bar{x})\right\}_{i \in I(\bar{x})} \cap-\operatorname{int} N(C ; \bar{x})$. Accordingly, there exist $\lambda_{i} \geq 0(i \in I(\bar{x}))$ with $\sum_{i \in I(\bar{x})} \lambda_{i}=1$ as well as $\varepsilon>0$ such that

$$
x^{*}=\sum_{i \in I(\bar{x})} \lambda_{i} \nabla f_{i}(\bar{x}) \quad \text { and } \quad \varepsilon\|x-\bar{x}\| \leq\left\langle x^{*}, x-\bar{x}\right\rangle \quad \forall x \in C .
$$

Due to the differentiability assumption on $f$ and to the finiteness of $I(\bar{x})$, there is some $\eta>0$ such that

$$
f_{i}(x)-f_{i}(\bar{x}) \geq\left\langle\nabla f_{i}(\bar{x}), x-\bar{x}\right\rangle-\frac{\varepsilon}{2}\|x-\bar{x}\| \quad \forall x \in B(\bar{x}, \eta), \forall i \in I(\bar{x}) .
$$

Using the fact that $f_{i}(\bar{x})=0$ for $i \in I(\bar{x})$, it holds for all $x \in C \cap B(\bar{x}, \eta)$ that

$$
\max _{i \in I(\bar{x})} f_{i}(x) \geq \sum_{i \in I(\bar{x})} \lambda_{i} f_{i}(x) \geq \sum_{i \in I(\bar{x})} \lambda_{i}\left\langle\nabla f_{i}(\bar{x}), x-\bar{x}\right\rangle-\frac{\varepsilon}{2}\|x-\bar{x}\| \geq \frac{\varepsilon}{2}\|x-\bar{x}\| .
$$

Measuring, without loss of generality, the distance in $\mathbb{R}^{m}$ with respect to the maximum norm, one has for all $x \in M(y) \cap B(\bar{x}, \eta)$ and all $y \in \mathbb{R}^{m}$

$$
d(x, M(0)) \leq\|x-\bar{x}\| \leq \frac{2}{\varepsilon} \max _{i \in I(\bar{x})} f_{i}(x) \leq \frac{2}{\varepsilon} \max _{i=1, \ldots, m}\left|y_{i}\right|=\frac{2}{\varepsilon} d(y, 0) .
$$

This, however, is the calmness of $M$ at $(0, \bar{x})$.

The last result shows that the ideas of the completely convex case can be extended to differentiable inequalities. With a single inequality which is differentiable and convex, (5.1) reduces to (3.4) (without the need of the additional constraint qualification $\left.\left(\mathrm{CQ}^{*}\right)\right)$. One might ask about an alternative condition in the sense of (3.3) for the 
differentiable case as well. However, the closedness of the normal cone immediately provides that the differentiable formulation of (3.3) implies (3.4); hence the two conditions are not independent as in the convex (nonsmooth) setting. Finally, we note that for finite-dimensional $X,(5.1)$ can be weakened to the condition

$$
\text { bd conv }\left\{\nabla f_{i}(\bar{x})\right\}_{i \in I(\bar{x})} \cap-\text { bd } N(C ; \bar{x})=\emptyset
$$

(see [7, Theorem 9]). In infinite dimensions, the interior of the convex hull involved is empty; hence this last relation is equivalent to (5.1).

6. Conclusion. The dual conditions for calmness formulated in this paper (in particular, (3.3), (3.4), (3.10), and (5.1)) are weaker than the usual Slater-type characterizations, which ensure the stronger Aubin property (or metric regularity) of the considered systems. These conditions can be immediately applied to various issues in mathematical programming such as error bounds, optimality conditions, weak sharp minima (see also [8]), or the stability of solutions under perturbations.

Acknowledgments. This paper originated from the authors' mutual visits at the University of Bourgogne, Dijon, and Weierstrass Institute Berlin, respectively. The authors express their gratitude to both institutions for their support.

\section{REFERENCES}

[1] J.-P. Aubin and I. Ekeland, Applied Nonlinear Analysis, Wiley, New York, 1984.

[2] J.M. Borwein And H.M. Strojwas, Tangential approximations, Nonlinear Anal., 9 (1985), pp. 1347-1366.

[3] J.M. Borwein AND D.M. ZhUANG, Verifiable necessary and sufficient conditions for regularity of set-valued and single-valued maps, J. Math. Anal. Appl., 134 (1988), pp. 441-459.

[4] F. Clarke, Optimization and Nonsmooth Analysis, Wiley, New York, 1983.

[5] S. Deng, Global error bounds for convex inequality systems in Banach spaces, SIAM J. Control. Optim., 36 (1998), pp. 1240-1249.

[6] R. Henrion, The Approximate Subdifferential and Parametric Optimization, Habilitation Thesis, Humboldt University, Berlin, 1997.

[7] R. Henrion and J. Outrata, A subdifferential criterion for calmness of multifunctions, J. Math. Anal. Appl., 258 (2001), pp. 110-130.

[8] R. Henrion, A. Jourani And J. Outrata, On the calmness of a class of multifunctions, SiAM J. Optim., 13 (2002), pp. 603-618.

[9] A.J. King AND R.T. RockAfEllar, Sensitivity analysis for nonsmooth generalized equations, Math. Programming, 55 (1992), pp. 193-212.

[10] A.B. LEVY, Implicit multifunction theorems for the sensitivity analysis of variational conditions, Math. Programming, 74 (1996), pp. 333-350.

[11] A. Jourani, Intersection formulae and the marginal function in Banach spaces, J. Math. Anal. Appl., 192 (1995), pp. 867-891.

[12] A. Jourani, Hoffman's error bound, local controllability, and sensitivity analysis, SIAM J. Control. Optim., 38 (2000), pp. 947-970.

[13] A. Jourani and L. Thibault, Metric regularity for strongly compactly Lipschitzian mappings, Nonlinear Anal., 24 (1995), pp. 229-240.

[14] D. Klatte and W. Li, Asymptotic constraint qualifications and global error bounds for convex inequalities, Math. Program., 84 (1999), pp. 137-160.

[15] A.S. Lewis AND J.-S. PANG, Error bounds for convex inequality systems, in Generalized Convexity, Generalized Monotonicity: Recent Results, J.P. Crouzeix, J.E. Martinez-Legaz, and M. Volle, eds., Kluwer, Dordrecht, the Netherlands, 1997, pp. 75-100.

[16] W. LI, Abadie's constraint qualification, metric regularity, and error bounds for differentiable convex inequalities, SIAM J. Optim., 7 (1997), pp. 966-978.

[17] W. Li And I. Singer, Global error bounds for convex multifunctions and applications, Math. Oper. Res., 23 (1998), pp. 443-462.

[18] B.S. Mordukhovich, Complete characterization of openness, metric regularity and Lipschitzian properties of multifunctions, Trans. Amer. Math. Soc., 340 (1993), pp. 1-35. 
[19] J.-S. PANG, Error bounds in mathematical programming, Math. Programming, 79 (1997), pp. 299-332.

[20] J.-P. Penot, Metric regularity, openness and Lipschitzian behavior of multifunctions, Nonlinear Anal., 13 (1989), pp. 629-643.

[21] S.M. Robinson, Normed convex processes, Trans. Amer. Math. Soc., 174 (1972), pp. 127-140.

[22] S.M. Robinson, Regularity and stability for convex multivalued functions, Math. Oper. Res., 1 (1976), pp. 130-143.

[23] S.M. Robinson, Some continuity properties of polyhedral multifunctions, Math. Program. Studies, 14 (1981), pp. 206-214.

[24] R.T. Rockafellar and R.J-B. Wets, Variational Analysis, Springer, New York, 1997.

[25] C. Ursescu, Multifunctions with closed, convex graph, Czechoslovak Math. J., 25 (1975), pp. $438-441$. 